\title{
Ocular Fundus Abnormalities Detected by Fluorescein and Indocyanine Green Angiography in the Royal College of Surgeons Dystrophic Rat
}

\author{
Takeo SATOH and Katsuhiro YAMAGUCHI \\ Department of Ophthalmology, Yamagata University School of Medicine, \\ 2-2-2 Iidanishi, Yamagata 990-9585, Japan
}

\begin{abstract}
The ocular fundi of the Royal College of Surgeons (RCS) dystrophic rats were examined by conventional fundus photography, fluorescein angiography (FA) and indocyanine green angiography (IA). In the fundus, a reddish colored background was observed in the RCS dystrophic rats at 3 weeks of age. At 9 weeks of age, the background had changed to pale in color. In FA, the RCS dystrophic rats at 3 week of age demonstrated background fluorescence with homogeneous brightness. Fluorescent dye leakage was observed in the late phase of the postinjection period at 9 weeks of age. In $I A$, the RCS dystrophic rats at 3 weeks of age had background fluorescence with homogeneous brightness, and at 5 weeks of age, spots of hyperfluorescence were scattered over the dark background. At 7 weeks of age, numerous delimited, irregular round spots of hyperfluorescence appeared over the dark background. Such hyperfluorescent lesions had further increased in number and size in the RCS dystrophic rats at 9 weeks of age. In this way, ocular findings related to abnormalities in the retinal pigment epithelium and choroid in the RCS dystrophic rat were demonstrated by fundus photography, fluorescein angiography and indocyanine green angiography.

Key words: fluorescein angiography, indocyanine green angiography, retinal degeneration, retinal pigment epithelium, Royal College of Surgeons dystrophic rat
\end{abstract}

\section{Introduction}

The Royal College of Surgeons (RCS) dystrophic rat strain is characterized by progressive degeneration of the photoreceptor cells [1,2]. A defect in the function of the retinal pigment epithelium (RPE), which has an intimate structural and functional relationship with the photoreceptor cell, has been shown to be the cause of a
99\% reduction in the number of photoreceptors in the outer nuclear layer by 2 to 3 months after birth. In addition to degeneration of photoreceptor cells, changes in the retinal vasculature have been described in this animal model for retinal degeneration by means of intravenous histochemical demonstration with horseradish peroxidase [3, 4], but there is little information on the fundus or angiographic analysis with the RCS dystro-

(Received 6 October 1999 / Accepted 14 April 2000)

Address corresponding: T. Satoh, Department of Ophthalmology, Yamagata University School of Medicine, 2-2-2 Iidanishi, Yamagata 9909585, Japan 
phic rat. We have examined the ocular fundus of the RCS dystrophic rat with a fundus camera and conducted fluorescein (FA) and indocyanine green (ICG) angiography (IA) with a scanning laser ophthalmoscope. FA is a useful diagnostic technique for retinal disorders. In contrast, IA allows enhanced imaging of choroidal abnormalities, as indocyanine green dye has high affinity for protein and therefore stays within the choroidal vasculature.

\section{Materials and Methods}

RCS dystrophic rats were obtained from CLEA (Japan). Controls in this study were age matched WKY rats (purchased from CLEA, Japan). At least 5 rats in each group were examined at 3,5,7, and 9 weeks of age. All the rats were maintained in a 12-hr light / 12$\mathrm{hr}$ dark cycle provided by cool-white fluorescent lights at an intensity of $6 \mathrm{ft}-\mathrm{c}$ at the cage level. All the rats were maintained and treated in accordance with NIH guidelines. They were anesthetized with sodium pentobarbital $(2 \mathrm{mg} / 100 \mathrm{~g})$ which was injected peritoneally, and their pupils were dilated with instillation of tropicamide and phenylephrine eyedrops. The ocular fundus was observed with a fundus camera (RC-2 Model 621, Kowa, Japan). Fundus photographs were taken with color film (Fuji ISO 100, Japan). FA and IA were performed with a scanning laser ophthalmoscope (SLO, Rodenstock, Germany) after dye was injected intravenously. For FA, the dose volume of $10 \%$ fluorescein dye was $0.03 \mathrm{ml} / 100 \mathrm{~g}$. For IA, the dose volume of ICG $(25 \mathrm{mg} / 2 \mathrm{ml})$ dye was $0.06 \mathrm{~m} / / 100 \mathrm{~g}$.

\section{Results}

In the fundus of a RCS dystrophic rat at 3 weeks of age, well-developed retinal arteriole and venules were distributed radially over the reddish colored background (Fig. 1A), but at 7 weeks of age the background had changed to a pale color (Fig. 1B), and at 9 weeks of age, the ocular fundus was much paler and the retinal vasculature became attenuated (Fig. 1C). In contrast, no abnormal changes were seen in the control WKY rats through 9 weeks of age (Fig. 1D).

In FA, the RCS dystrophic rats at 3 weeks of age demonstrated a bright background fluorescence that appeared gradually over the time course, and the background fluorescence was uniformly bright by $5 \mathrm{~min}$ after dye injection (Fig. 2A). But in RCS dystrophic rats at 5 weeks of age, hyperfluorescent dye leaked at numerous sites over the bright background by $5 \mathrm{~min}$ after dye injection (Fig. 2B). At 7 weeks of age, the leakage of fluorescent dye spread over the dark background by $5 \mathrm{~min}$ after dye injection (Fig. 2C). The fluorescent dye leakage was observed from the early phase, and remarkably increased in the late phase over 5 min after dye injection in RCS dystrophic rats at 9 weeks of age, but in the control WKY rats, no such fluorescein leakage was seen through 9 weeks of age (Fig. 2D).

IA in RCS dystrophic rats clearly demonstrated choroidal and retinal circulation early in the postinjection period. At 3 weeks of age, bright background fluorescence appeared gradually with time by 5 min after dye injection (Fig. 3A), but at 5 weeks of age numerous spots of hyperfluorescence had scattered over the dark background by $5 \mathrm{~min}$ after dye injection (Fig. 3B). And at 7 weeks of age, hyperfluorescent areas appeared as numerous irregular round spots over the dark background by 5 min after dye injection. Some of these spots were located beneath the retinal vessels (Fig. 3C). Such hyperfluorescent spots had further increased in number and size in RCS dystrophic rats at 9 weeks of age (Fig. 3D). In control WKY rats, the choroidal and retinal circulations were clearly observed early in the IA, and following the time course, bright background fluorescence gradually appeared. The background fluorescence was uniformly bright by $5 \mathrm{~min}$ after dye injection (Fig. 3E) as was seen in RCS dystrophic rats at 3 weeks of age (Fig. 3A).

\section{Discussion}

In this study, a fundus abnormality in RCS dystrophic rats was detected by means of a conventional fundus camera, and fluorescein and Indocyanine green angiography for the first time to our knowledge. With a conventional fundus camera, well-developed retinal arterioles and venules were observed radially over the reddish-colored background in RCS dystrophic rats at 3 weeks of age. In FA, the background fluorescence was uniformly bright late in the postinjection period. In IA, choroidal and retinal circulations were clearly observed, and the background fluorescence was uni- 

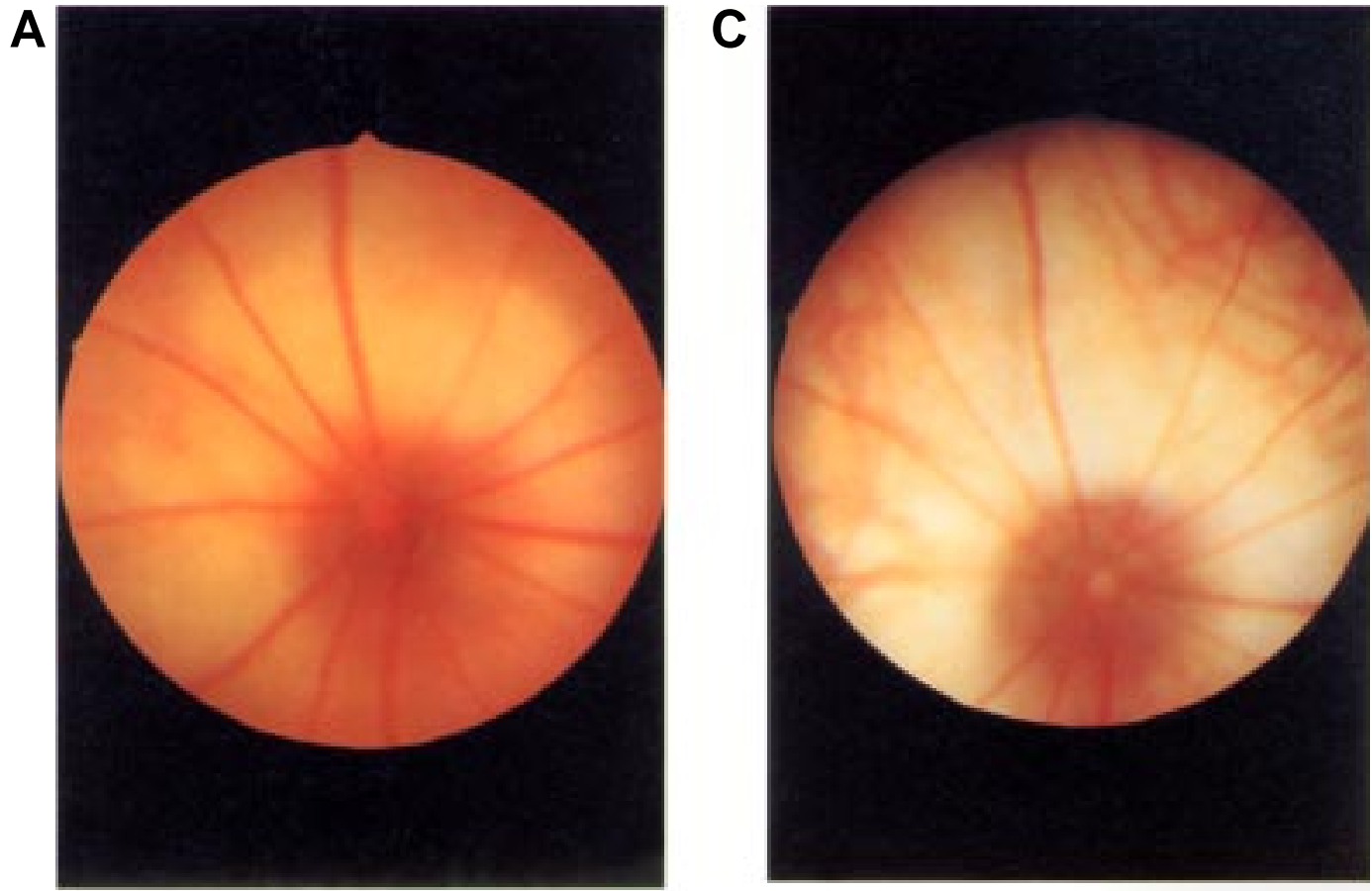

B

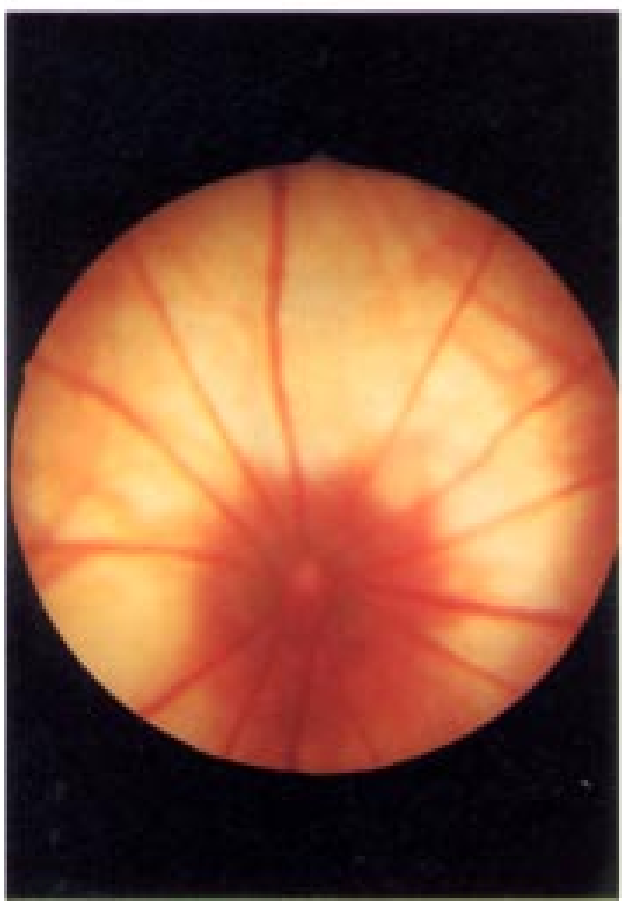

D

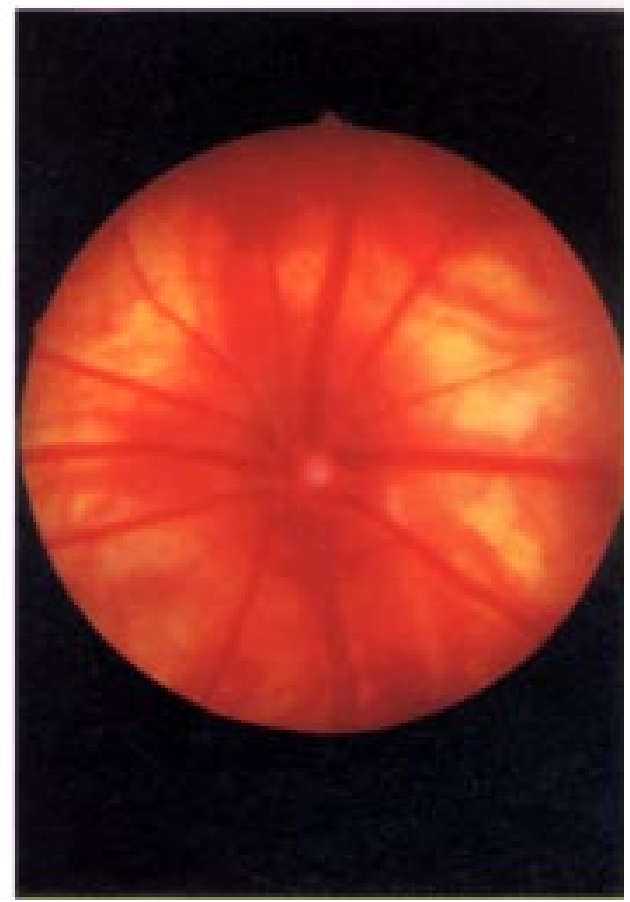

Fig. 1. Fundus photograph. (A) RCS dystrophic rat at 3 weeks of age: Well-developed retinal arterioles and venules are seen over the reddish colored background. (B) RCS dystrophic rat at 7 weeks of age: The background has changed to pale color. (C) RCS dystrophic rat at 9 weeks of age: The ocular fundus is much paler and the retinal vasculature is attenuated. (D) Control WKY rat at 9 weeks of age: No fundus abnormality is seen. 

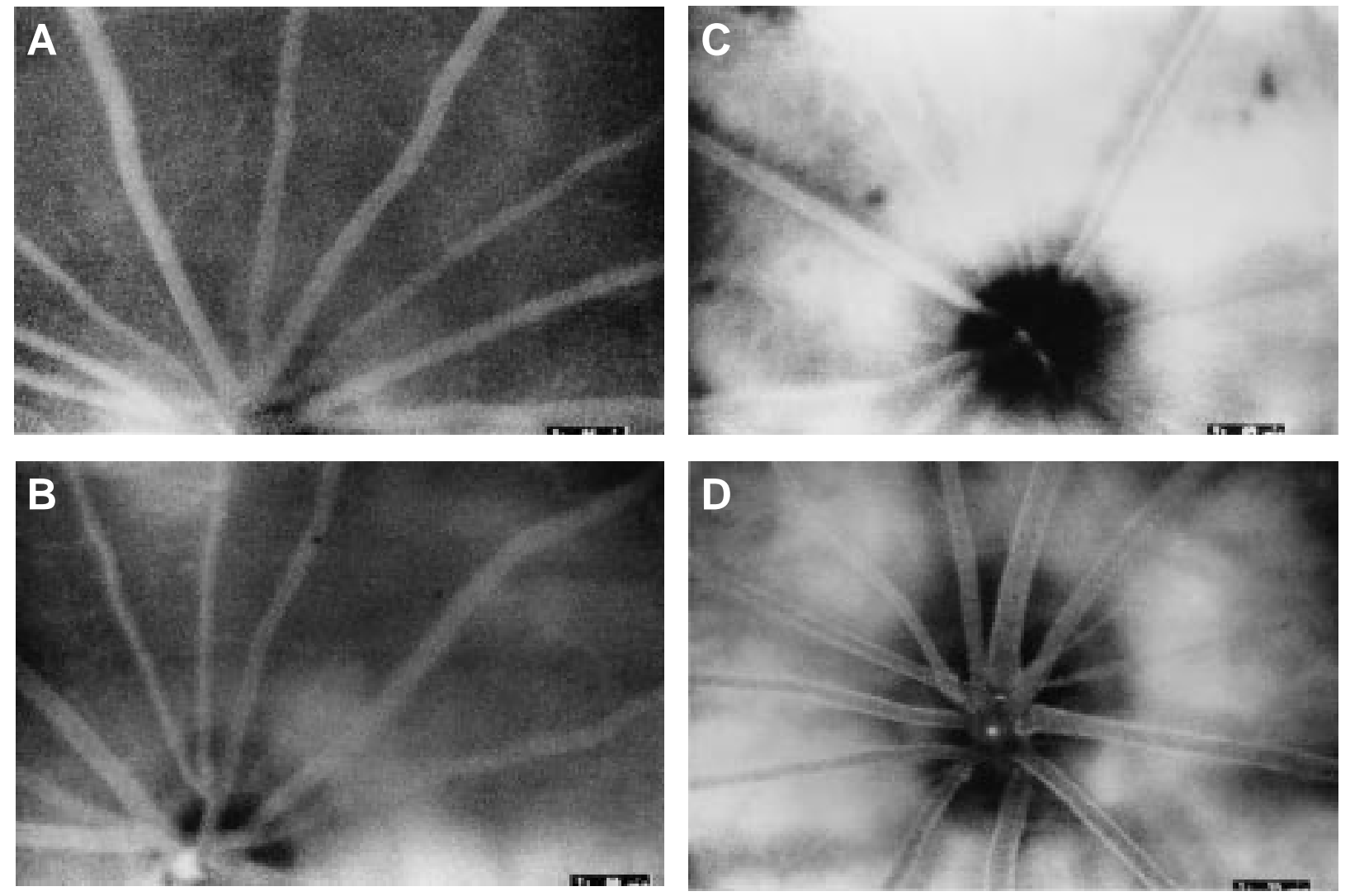

Fig. 2. Fluorescein angiography. (A) RCS dystrophic rat at 3 weeks of age: The background fluorescence is uniformly bright. (B) RCS dystrophic rat at 5 weeks of age: Hyperfluorescent dye leaked over the bright background. (C) RCS dystrophic rat at 7 weeks of age: Fluorescent dye leakage appears over the background. (D) Control WKY rat at 9 weeks of age: No fluorescein leakage is seen.

formly bright in the early and late phases of the postinjection period respectively. Similar findings were seen in WKY rats from 3 to 9 weeks of age, indicating that these findings were normal characteristics of the fundus.

In contrast, the background of the fundus changed to pale color in the RCS dystrophic rats at 9 weeks of age. FA showed dye leakage late in the postinjection period. A breakdown in maintaining permeability of the RPE seemed to play a role in dye leakage observed by FA. In RCS dystrophic rats, the retina develops normally until 3 weeks of age, after which time photoreceptor cells degenerate progressively, and as a result the outer nuclear layer becomes thinner. Since the RPE cells of RCS dystrophic rats have a defective phagocytosis of shed rod outer segments, the debris of the outer segment begins and continues to accumulate at the surface of the RPE until 2 months of age [5-7]. The RPE cells begin to appear to have an abnormally flattened or a convoluted surface as morphological changes indicative of their dysfunction, so that in the RCS dystrophic rats the leakage of the fluorescent dye from the choroid was shown to be the result of dissolution of tight junctions between RPE cells $[8,9]$. The severity of the dissolution of tight junctions could reflect the intensity of the hyperfluorescence of the fundus as shown in Fig. 2 in this paper.

In addition, IA enabled us to examine the abnormality in detail after 5 weeks of age. The background fluorescence of RCS dystrophic rats was darker than that of the control rats and became progressively darker with age. Caldwell and co-investigators reported that the choriocapillaris was usually absent, and the choroid was substantially thinner than normal in the areas near large vascular vitreoretinal membranes in RCS dystrophic rats [10]. As the choriocapillaris provides metabolic support 

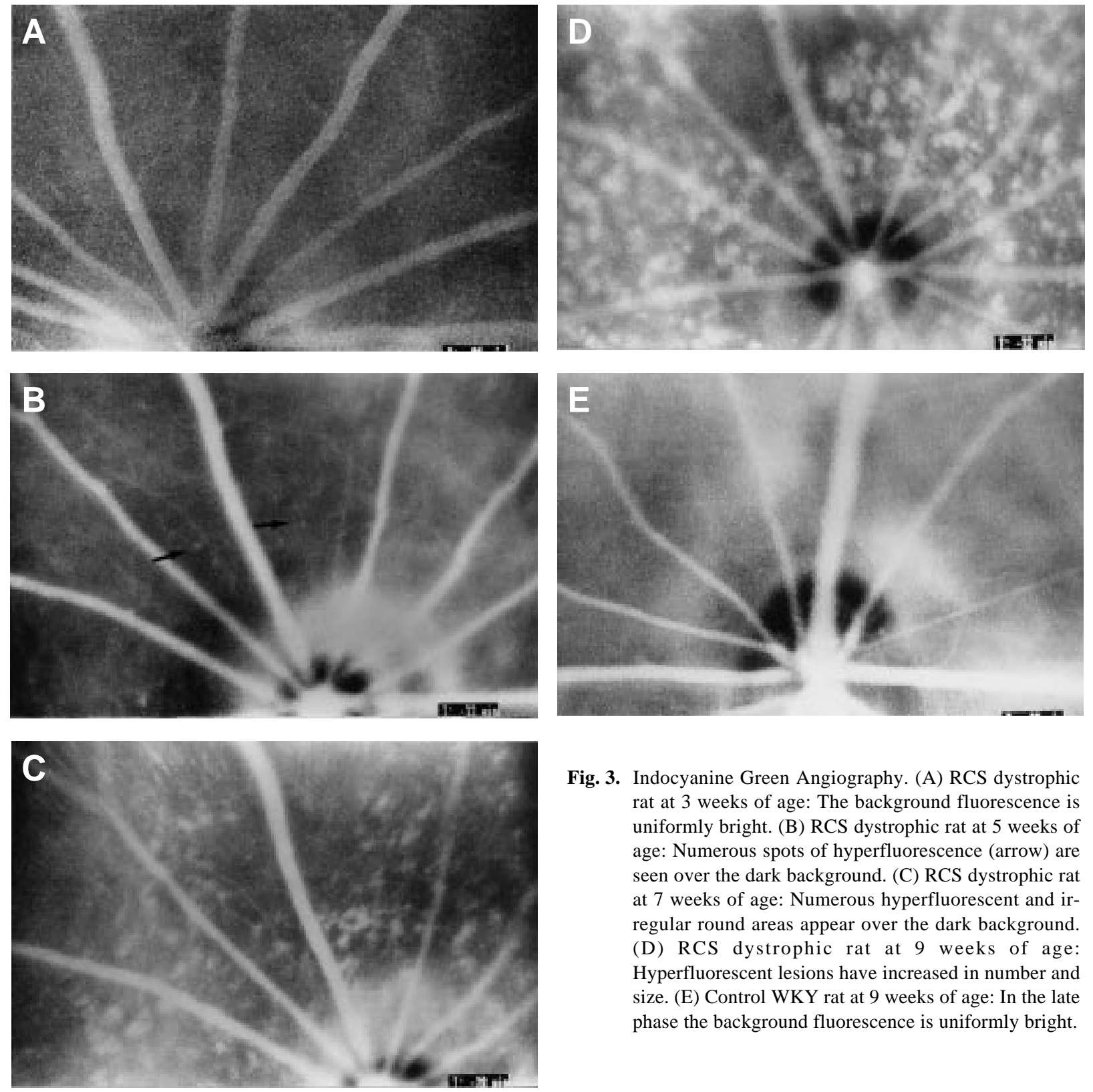

Fig. 3. Indocyanine Green Angiography. (A) RCS dystrophic rat at 3 weeks of age: The background fluorescence is uniformly bright. (B) RCS dystrophic rat at 5 weeks of age: Numerous spots of hyperfluorescence (arrow) are seen over the dark background. (C) RCS dystrophic rat at 7 weeks of age: Numerous hyperfluorescent and irregular round areas appear over the dark background. (D) RCS dystrophic rat at 9 weeks of age: Hyperfluorescent lesions have increased in number and size. (E) Control WKY rat at 9 weeks of age: In the late phase the background fluorescence is uniformly bright.

to the outer neurosensory retina and RPE, an intimate relationship between the RPE and choroidal vasculature is of considerable importance in ocular disease. Sarks has pointed out a correlation between RPE dropout and atrophy of the choriocapillaris in the corresponding areas in humans indicating a definite relationship between the RPE and choriocapillaris [11]. The dark area we detected in IA could be explained as the area of atrophy of the choriocapillaris in RCS dystrophic rats. Our results therefore indicate that atrophy of the choriocapillaris is closely linked to the progressive retinal degeneration in RCS dystrophic rats and will contribute to understanding the mechanism of this dystrophic disease.

The dark area and bright patches resulted in a mosaic appearance in the IA images of RCS dystrophic rats. With regard to the numerous hyperfluorescent patches, they increased in intensity and size throughout the time course and the maximal lesions were attained by $5 \mathrm{~min}$ postinjection. Since some of them were observed beneath the retinal vessels, these hyperfluorescent lesions 
were considered to exist in the RPE layer or in the choroid. The number and size of the lesions increased with age. It was hypothesized that ICG molecules might accumulate within the subretinal debris which could be a target for ICG molecule binding [12]. The increases in both the intensity of hyperfluorescence and the size of the lesion with age were consistent with the finding that the debris continues to accumulate at 2 months of age. Discontinuous atrophy of the choriocapillaris and accumulation of ICG molecules into the subretinal debris appeared to contribute to the formation of the mosaic pattern characterized by dark and bright patches in RCS dystrophic rats $[13,14]$. The findings obtained in this study were considered to be closely related to the retinal degeneration in this animal model. Our study visualized the fundus abnormality in RCS dystrophic rat, adding a new area of interest to the study of this animal model of retinal degeneration.

\section{Acknowledgments}

The authors thank Dr. Kazuo Ohwada, Laboratory Animal Center, Dr. Yoshinori Takahashi, Dr. Ryo Kawasaki, and Takashi Yamamoto of the Department of Ophthalmology for generous assistance, and Professor Emeritus of Department of Ophthalmology Shigeki Takahashi for helpful advice.

\section{References}

1. Dowling, J.E. and Sidman, R.L. 1962. Inherited retinal dystrophy in the rat. J. Cell Biol. 14: 73-109.

2. LaVail, M.M., Sidman, R.L., and Gerhardt, C.O. 1975. Congenic strains of RCS rats with inherited retinal dystrophy. J. Hered. 66: 242-244.

3. Mattes, M.T. and Bok, D. 1985. Blood vascular abnormalities in animals with inherited retinal degeneration. pp. 209-237. In: Retinal Degeneration; Experimental and Clinical Studies. (LaVail, M.M., Hollyfield, J.G., and Anderson, R.E. eds.), Alan R. Liss Inc., New York.

4. Seaton, A.D. and Turner, J.E. 1992. RPE transplants stabilize retinal vasculature and prevent neovascularization in the RCS rat. Invest. Ophthalmol. Vis. Sci. 33: 83-91.

5. Bok, D. and Hall, M.O. 1971. The role of the pigment epithelium in the etiology of inherited retinal dystrophy in the rat. J. Cell Biol. 49: 664-682.

6. LaVail, M.M., Sidman, R.L., and O'Neil, D. 1972. Photoreceptor-pigment epithelial cell relationships in rats with inherited retinal degeneration; Radioautographic and electron microscope evidence for a dual source of extralamellar material. J. Cell Biol. 53: 185-209.

7. Tamai, M. and O'Brien, P.J. 1979. Retinal dystrophy in the RCS rat: in vivo and vitro studies of phagocytic action of the pigment epithelium on the shed rod outer segments. Exp. Eye. Res. 28: 399-411.

8. Caldwell, R.B., McLaughlin, B.J., and Boykins, L.G. 1982. Intramembrane changes in retinal pigment epithelial cell junctions of the dystrophic rat retina. Invest. Ophthalmol. Vis. Sci. 23: 305-318.

9. Caldwell, R.B. and McLaughlin, B.J. 1983. Permeability of retinal pigment epithelial cell junctions in the dystrophic rat retina. Exp. Eye Res. 36: 415-427.

10. Caldwell, R.B., Roque, R.S., and Solomon, S.W. 1989. Increased vascular density and vitreoretinal membranes accompany vascularization of the pigment epithelium in the dystrophic rat retina. Curr. Eye Res. 8: 923-927.

11. Sarks, S.H. 1990. Drusen and their relationship to senile macular degeneration. Aust. J. Ophthalmol. 8: 117-130.

12. Parodi, M.B., Instulin, D., Russo, D., and Ravalico, G. 1996. Adult onset foveomacular vitelliform dystrophy and indocyanine green videoangiography. Graefe's Arch. Clin. Exp. Ophthalmol. 234: 208-211.

13. Guyer, D.R., Puliafito, C.A., Mones, J.M., Friedman, E., Chang, W., and Verdooner, S.R. 1992. Digital indocyanine green angiography in chorioretinal disorders. Ophthalmology 99: 287-291.

14. Bartsch, D.U., Weireb, R.N., Zinser, G., and Freeman, W.R. 1995. Confocal scanning infrared laser ophthalmoscopy for indocyanine green angiography. Am. J. Ophthalmol. 120: 642-651. 